\title{
Mycophenolate Mofetil
}

National Cancer Institute

\section{Source}

National Cancer Institute. Mycophenolate Mofetil. NCI Thesaurus. Code C1468.

The morpholinoethyl ester of mycophenolic acid (MPA) with potent immunosuppressive properties. Mycophenolate stops T-cell and B-cell proliferation through selective inhibition of the de novo pathway of purine biosynthesis. In vivo, the active metabolite, MPA, reversibly inhibits inosine 5'-monophosphate dehydrogenase, an enzyme involved in the de novo synthesis of guanine nucleotides. MPA displays high lymphocyte specificity and cytotoxicity due to the higher dependence of activated lymphocytes on both salvage and de novo synthesis of guanine nucleotides relative to other cell types. ( $\mathrm{NCI04)}$ 